\section{Public Health} Genomics
Public Health Genomics 2012;15:352-362

DOI: $\underline{10.1159 / 000343792}$
Received: June 1, 2012

Accepted after revision: September 24, 2012

Published online: November 15, 2012

\title{
Experiences of Early Users of Direct-to-Consumer Genomics in Switzerland: An Exploratory Study
}

\author{
E. Vayena ${ }^{\text {a }} \quad$ E. Gourna ${ }^{\text {a }} \quad$ J. Streulia ${ }^{a}$ E. Hafen ${ }^{\text {b }}$ B. Prainsack ${ }^{c}$ \\ ${ }^{a}$ Institute of Biomedical Ethics, University of Zurich, and ${ }^{b}$ Institute for Molecular Systems Biology, ETH Zurich, \\ Zurich, Switzerland; ' Department of Sociology and Communications, School of Social Sciences, Brunel University, \\ Brunel, UK
}

\section{Key Words}

Direct-to consumer · Early users · Personal genomics •

Personal utility $\cdot$ Switzerland $\cdot 23$ andMe

\begin{abstract}
Aims: This study explores attitudes, motivations and self-reported impact in connection with direct-to-consumer (DTC) genomic testing amongst a group of life scientists in Switzerland. Methods: Data were collected through: (1) a self-completion online questionnaire, and (2) semi-structured qualitative interviews. Forty participants completed the questionnaire and 10 were interviewed. Results: Curiosity was mentioned as the primary reason for undergoing testing, while less significance was attributed to receiving actionable health information. The opportunity to contribute to research ranked high as a motive for testing. Overall, participants assessed their experience with the test as positive and were willing to recommend it to others. Some reported that the testing had an impact on how they view their health, but only a third of participants planned on showing the results to health practitioners. Participants consistently referred to 'fun' when describing several aspects of the testing experience. The 'fun factor' manifested itself in different phases of the process, including the motivation for taking the test, receiving the information and putting the test results to use
\end{abstract}

(including sharing and discussing it with others). This finding suggests the need to further explore the concept of personal utility in DTC genomics. Conclusions: Although this group is not representative of the broader population due to both their scientific expertise and their willingness to try out a controversial new technology, their experiences provide valuable insights into the role of personal curiosity and altruism (fostering medical research) as motivations for testing and the utility attributed to both.

Copyright $\odot 2012$ S. Karger AG, Basel

\section{Introduction}

For a long time, genetic information for health purposes was obtained in clinical settings only, prescribed by a physician on the basis of a specific indication, and with pre- and post-test genetic counselling. Since the end of the last decade, consumers have been able to obtain information about their genetic make-up (traits, ancestry information, disease susceptibility, etc.) directly from commercial companies. These companies are mostly located in the USA (e.g. 23andMe) or in Europe (e.g. deCODEme in Iceland and biologis.com in Germany). Most companies use single nucleotide polymorphism (SNP) genotyping technology to generate their results.

\section{KARGER \\ Fax +4161306 1234 \\ E-Mail karger@karger.ch}

www.karger.com
(C) 2012 S. Karger AG, Basel

$1662-4246 / 12 / 0156-0352 \$ 38.00 / 0$

Accessible online at:

www.karger.com/phg
Effy Vayena, $\mathrm{PhD}$

Institute of Biomedical Ethics, University of Zurich

Pestalozzistrasse 24

$\mathrm{CH}-8032$ Zurich (Switzerland)

E-Mail vayena@ethik.uzh.ch 
Because tests are ordered and results received online, national borders are regularly crossed.

In the context of movements such as 'Quantified Self' (http://quantifiedself.com/) and other initiatives that harness the potential of individually generated and annotated data, infrastructures that facilitate individuals' access to their personal genomic information (and possibly links with other relevant datasets) could reap benefits for personalised medicine $[1,2]$. The debate about personal genomics and its role in the emergence of personalised medicine, however, has been overshadowed by the controversy about the risks and benefits of the directto-consumer (DTC) model. The controversy has to a large extent become locked in polarised positions [3], with the central point of critique of the opponents of DTC genomics being its low predictive value, as well as its allegedly non-existent, or very low, clinical validity and clinical utility of the information provided by DTC genomics. Key to this argument is the claim that the information is not medically actionable $[4,5]$. Furthermore, opponents of DTC genomics argue that such information provided without genetic counselling is likely to be misunderstood by individuals and thus liable to cause harm, e.g. fostering false confidence about not being at risk for a disease or causing unwarranted anxiety [6,7]. Moreover, worried consumers might undergo unnecessary and costly medical tests, raising health-care costs without any benefit [8]. Concerns about privacy of genomic data have also been central to the debate [9]. On the other side of the divide, proponents of DTC genomics argue that such information, which is not intended for medical diagnosis, empowers consumers. If individuals better understand the genomic underpinnings of diseases and traits, they will be able to make more informed decisions about their lives and their lifestyles. In general, they will be more informed participants in, and recipients of, health care. The criticism regarding the questionable validity and utility of the information provided by DTC genomics is countered by some of the proponents of PG genomics by reference to other services that we routinely use in our daily lives and that have not spurred significant debate. Such services are legal with the proviso of caveat emptor (i.e. the assumption that the customer should know what she is buying), and thus, so the argument goes, DTC genomics companies should be treated in the same manner [10, 11]. Moreover, despite their agreement on the low clinical utility of the information overall, proponents of DTC genomics argue that some sub-sets of the results provided by companies do have immediate clinical value: information on drug metabolism, for example, could have tangible ben-

Early Users of Direct-to-Consumer

Genomics in Switzerland efits for the test-taker, e.g., if one knows that one is sensitive to warfarin, one can use this information for dose adaptation [12].

While there is merit in most of these arguments, what became quickly obvious was the dearth of evidence as to why consumers might (or might not) undertake such testing, what such tests mean to them, what impact they have on them, and why. Such evidence is, however, critical in informing not only the normative arguments about the moral acceptability of DTC but also the discussion about suitable regulatory approaches.

Several studies have been conducted to explore these questions [13]. The majority of studies which examined attitudes and impact of DTC genomic testing have been conducted in the USA [13]. They concerned early adopters and focused on motivation for undergoing testing, understanding of risk and impact of test results. Some of the studies included large samples as parts of ongoing projects [14]. Studies outside the North American continent include quantitative and qualitative analyses of attitudes towards DTC genomics; however, to the best of our knowledge, they have not focused on consumers residing outside North America who have already undertaken DTC testing. One study analysed blog entries posted on DTC genomic websites without distinguishing national origin [15]. In sum, there has been no study large and robust enough to allow generalisations, especially not to publics outside of North America.

Because commercial genomics first appeared in the North American market and operated in English, advertising has probably been most effective in this region as well. This assumption is supported by findings that at the peak of the media coverage on DTC genomics in the middle of 2008 , only $13 \%$ out of 4,050 adults in survey in the UK had heard of such services [16], indicating that awareness was not very high even in English-speaking countries outside of North America (or possibly even only certain areas in the USA). In principle, however, commercial genomics services are available to customers all over the world. Furthermore, countries (or in the USA, certain states) that prohibit providers from offering DTC genetic and genomic testing can only do this on their own territory. Such laws are not binding on consumers of DTC services who are physically located in other jurisdictions (nevertheless, some DTC genomics companies voluntarily refrain from shipping to areas where DTC genetic and genomic testing is prohibited).

While the first companies offering genomics testing services DTC were 23andMe in Calif., USA, and deCODEme in Iceland, similar companies started ap- 
pearing on the European continent in the early 2010s, indicating that the phenomenon of consumer genomics might be searching for its niche in the European markets (see, for example, biologis.com). Although the future of consumer genomics will depend on several factors that are impossible to predict [17], it is important to start exploring consumers' expectations and understanding of, and satisfaction with, DTC genomics outside the North American context. Not only might there be national and cultural differences regarding motivations and perceptions, but there are also different regulatory frameworks governing such activities within European countries; some of them are distinctly different from North America [18].

Our study explores attitudes, motivations and self-reported impact in connection with DTC genomic testing amongst a group of life scientists in the Swiss Federal Institute of Technology (ETH) and the University of Zurich in Switzerland. Although the study was conducted in 2011, we use the term early users to refer to study participants, because notwithstanding that commercial genomics have been available online since 2007, levels of public awareness of the availability of the tests seem to have been limited in Switzerland. We base this assumption on the limited press coverage of DTC issues over the last few years as well as the limited search volume of related keywords in the Google search engine (we ascertained the latter by using GoogleInsights [19]). Search volumes above the threshold for presentation were only from the Zurich area, with 2 spikes: one around 2008 and the second after the summer of 2011. The first spike, in 2008, was very plausibly caused by the intense media interest in the launch of the first DTC genomics companies in the USA, which 'spilled over', if only on a much smaller scale, to continental Europe. The second spike is likely due to a well-publicised conference on genomic medicine in September 2011, organised by the Academia Engelberg Foundation whose mission is to foster interdisciplinary dialogue about social values and scientific progress [20]. Therefore, it is reasonable to assume that the participants in our study group were among the first in the country to have undergone DTC genomics testing.

An additional reason for the 'slow' uptake of DTC genomics testing in Switzerland may be that commercial DTC genomics companies, with the exception of the German service bio.logis.com which has been available only since 2011, operate in English. Although the general level of education in Switzerland is high [21], and most people have a good enough command of English to converse about everyday matters, fewer are expected to know En- glish well enough to engage with the technical and scientific language used by DTC genomics services [22].

All participants in this study reside in Switzerland, a country with one of the highest GDP and GDP per capita in the world. Both public and private health expenditure are comparatively high. Health insurance coverage is universal, and life expectancy is amongst the highest worldwide. Switzerland has a friendly tax and legal environment for business enterprises and a strong biotech and life science industry $[23,24]$. The legal environment for DTC genomics is less friendly: Swiss law (National Law on Genetic Examinations on Humans, Bundesgesetz über genetische Untersuchungen beim Menschen) prohibits genetic and genomic testing that is not prescribed by a physician for a medical indication [25]. Therefore, commercial genomic companies that offer direct-to-consumer services are prohibited from doing so from Swiss territory. As was discussed above, this law does not prohibit that individuals purchase such services abroad. In 2009, an expert genetics committee appointed by the Swiss Ministry of Health issued a recommendation advising the public to refrain from using genetic tests that are available online. The main reasons cited by the committee were the limited clinical validity and utility of such tests and concerns about personal data protection [26]. Switzerland already has strict data protection laws.

\section{Methodology}

As a part of graduate courses and seminars in molecular genetics at the ETH and the University of Zurich, one of the study authors and course instructors (E.H.) introduced the issue of commercial genomics (sequencing technology, clinical validity, links to personalised medicine) to the audience (researchers and students). At the end of each session, members of the audience who were interested in better acquainting themselves with genotyping were offered the opportunity to register their name with the speaker who would then make a bulk order (and bulk shipment) of 23andMe tests. For this purpose, a special educational rate was offered by 23 andMe (USD 99 instead of the standard USD 199 price at the time). A total of 60 people registered. An administrator at the Institute of Molecular Biology at the ETH coordinated the order with 23andMe.

Given the considerable interest in DTC genomics testing among the members of the audience, 2 authors of this paper (E.V. and E.H.) discussed the possibility of designing a study to explore the experience of these test-takers with DTC genomics. Permission from the research ethics review committee of the canton of Zurich was requested and received. We then started data collection through: (1) a self-completion online questionnaire, and (2) semi-structured qualitative interviews carried out in person or by phone. 
Table 1. Demographics of study participants

\begin{tabular}{lll}
\hline & $\begin{array}{l}\text { Questionnaire, \% } \\
(\mathrm{n}=60)\end{array}$ & $\begin{array}{l}\text { Interviews, \% } \\
(\mathrm{n}=10)\end{array}$ \\
\hline Response rate & $66.7(40)$ & $100(10)$ \\
Female & $34.2(13)$ & $70(7)$ \\
Male & $65.8(25)^{* *}$ & $30(3)$ \\
Bachelor's degree & $7.9(3)$ & 0 \\
MSc & $39.5(15)$ & $22.2(2)$ \\
PhD & $52.5(20)^{* *}$ & $77.8(7)^{*}$ \\
Mean age & - & 34.5 \\
Respondents have children & - & $50(5)$ \\
\hline
\end{tabular}

* 1 missing. ${ }^{* *} 2$ missing.
$[29,30]$. In parallel, 3 of the authors (E.G., J.S., E.V.) reviewed the interview transcripts and developed first level codes. Codes were developed on the basis of existing literature and preliminary findings from the quantitative data. First level codes were then collapsed into advanced categories. The frequency of codes was taken into account for the development of categories. Categories were analysed for the identification of key themes.

\section{Results}

\section{Results from the Questionnaires}

All those who filled in the online questionnaires had studied life science subjects, and most of them held graduate degrees: $8 \%$ had a Bachelor's degree, $40 \%$ a Master's degree and $52 \%$ had a $\mathrm{PhD} ; 66 \%$ of the questionnaire respondents were male. Due to the relatively small size of the group, we refrained from asking about age and nationality, to avoid the possibility of identification of some participants; however, as the group consisted predominantly of post-graduate students, we assume that this cohort was relatively young, with ages ranging between 25 and 40 (table 1). Although no data on nationality exists, we know that all respondents are residents in Switzerland.

When filling in the questionnaire, respondents were asked to choose all motivations for testing that applied to them from a list of 4 questions. Questions included the general usefulness of genetic information in relation to disease susceptibility, including information that can lead to preventative steps, curiosity, family history of genetic disease, and the option to give additional reasons. It was possible to choose more than one reason. Most respondents named curiosity as their main reason for undergoing the test. Interestingly, the usefulness of genetic information, especially as a basis for taking measures to prevent disease, was the second most common reason.

Nine out of 10 respondents described their experience with DTC genome testing as positive, and over 7 out of 10 (73\%) said they would recommend this test to a family member or a friend. Answers to questions about their satisfaction with the information that they had received indicated, overall, a positive perception. Most respondents (64\%) said they were likely to share the information with their families. When asked whether they deemed it acceptable that such tests were available online, based on their own experience with this service, 6 out of 10 answered affirmatively. More than half of the respondents said the results had an impact on how they thought about their health (55\%), while just over a third disagreed with 
this statement (35\%). Less than a third of the participants intended to show the results to a health practitioner $(28 \%)$, one-third were undecided (33\%), and a slightly larger group (38.5\%) were not planning to show the results to a physician.

More than half of the respondents (55\%) were not very concerned about privacy and confidentiality issues. Among those who did express concern, risks related to health insurance coverage were most frequently mentioned. When prompted to further specify their concerns, mention was made of third-party companies who may access their data and of data security issues.

Most respondents (66\%) also intended to participate in research that was offered by the genomics testing company in a separate section on their website ('23andWe'). Participating in research mostly consists in filling in questionnaires about lifestyle, health and selected phenotypes, thus enabling the company to link this information with the test-takers' SNP data [31]. Answers to our open-ended question about why participants would engage in this type of company-driven research showed that most respondents considered such research valuable, acknowledged the need for more data, welcomed an opportunity to contribute to the common good, and were encouraged by the fact that they could choose the type of research they would be involved with (table 2).

\section{Results from the Interviews}

All 10 participants who agreed to be interviewed had studied life science subjects. Seven were female and 3 were male. Seven had a PhD degree, 2 a Master's degree and were working towards their $\mathrm{PhD}$, and 1 informant had both a $\mathrm{PhD}$ and an MD degree. The mean age of our participants was 35 .

Our qualitative data summarised in table 3 suggest that this group was driven primarily by general curiosity to learn new things and to a lesser extent by a goal-oriented desire for personal health information. Seven of our 10 informants stated curiosity as the main reason for taking the test, as exemplified by the following quote:

Interviewer: 'If you had to name one main reason for taking the test, what would it be?'

Respondent (R) 2: 'Of course I was curious to know if there is a disease, if there is something you can do [something] about it, but for me it was more from a scientific point of view to see what would they find out, what they could tell. It was more scientific curiosity.'

Some respondents said explicitly that there was no specific question to which they expected the test to provide an answer. This is how $\mathrm{R} 5$ put it:
'[...] so there was no specific issue I wanted to know, just general overview on how they do it and how differently results are presented.'

Other themes that emerged from the analysis of the qualitative data on motivation for testing were the 'fun factor' (mentioned by 7 respondents), the opportunity to foster research (half of the respondents) and a distinction between scientific curiosity and general curiosity (4 respondents).

'[It felt like] just a cool idea to do it. Also to do the overall testing with so many people. An overwhelming mass of data. [...] I thought it was a good idea also because we need more studies, more data.' (R 9)

\section{Other respondents echoed this notion, such as $\mathrm{R} 10$ :}

'Plain curiosity was the main reason for me [...] and the second to provide my genome information to society for research purposes.'

The 2 notions - satisfying one's own curiosity, and helping research - were closely connected. This connection was also reflected in the open-ended questions of the survey questionnaire:

'Phenotype-genotype associations are key to the better understanding of genetic information. I feel that my genetic information coupled with my [phenotypic] features is something I can give to society.' (This comment was taken from an open-ended question in the questionnaire.)

Respondents reflecting on the testing experience described it as mostly positive, mainly because they found that the test satisfied their curiosity. Some indicated that they had not high expectations in relation to health information when taking the test and ranked the fact that the test had satisfied their curiosity and familiarised them with commercial genomics services above the importance of the information that is immediately relevant for their health. In the words of $\mathrm{R} 4$,

'I feel I gained experience in dealing with this sort of probabilistic data in relation to my health. It depends on what you mean by useful, but if you mean like something I can directly use in relation to medical care, I would say: [it did] little.'

This respondent in fact challenged the common understanding of the term 'utility' when used in the context of genetic testing. The term 'utility', when used in connection with DTC genomics tests, is typically used synonymously with clinical utility, signifying immediate or at least predictable actionability in a clinical context. For our respondents, however, utility was much broader: 
Table 2. Data from the survey questionnaires

Selected

Reasons for testing*

- Useful in general and specifically to improve health (through prevention, lifestyle changes)

$50 \quad 50$

- Curiosity

- Family history of genetic diseases

$88-12$

- Other reasons: (ancestry, drug response information, compare genetic profile with family members)

Experience with the test*

- The overall experience was positive

- The overall experience was indifferent

- The overall experience was negative

- I would recommend this test to a family member or friend

- The results make perfect sense to me and I feel I understand all of it

- The results make some sense to me and I feel I understand enough of it

- The results make very little sense to me and I do not feel I understand it

- The information was presented in a very clear and accessible manner

- I will need the help of a medical geneticist to understand the results

- I would like to share the results with my family

10

90

Given the experience with the test, how should such tests be allowed to be accessed by individuals?*

- Over the counter on the Internet

- By medical prescription only

- Under supervision/counseling at a health care facility

$\begin{array}{ll}90 & 10 \\ 8 & 92 \\ 2 & 97 \\ \text { Yes: } 73 & \text { No: } 5 \\ 33 & 67 \\ 61 & 39 \\ 5 & 95 \\ 72 & 28 \\ 18 & 82 \\ 64 & 15\end{array}$

Did the information you received impact on how you think of your health?*

- Some impact

- Serious impact

- No impact

- Do you intend to show your results to your doctor?

$\begin{array}{rr}60 & 40 \\ 2 & 98 \\ 38 & 62\end{array}$

Concerns about DTC testing*

- Serious concerns about privacy and confidentiality $\quad 2 \quad 98$

- Some concerns about privacy and confidentiality

- No concerns at all

- Concerns about health insurance

- Concerns about future employment

- Concerns use of my data for research that I disagree with

- Concerns about access by third-party companies, data safety

- Intention to participate in surveys and research

$\begin{array}{ll}55 & 45 \\ 10 & 90 \\ 35 & 65 \\ \text { Yes: } 28 & \text { No: } 39\end{array}$

The table gives information on the percentage of respondents who chose an option (1st column) versus those who did not choose it ( 2 nd column), and who agreed with a statement (1st column) versus those who disagreed (2nd column). The remaining percentages include those who did not answer or chose 'I do not know'.

* Multiple options from the list below could be selected.

'.. and then I was particularly interested in the traits, in fact what you can actually see from this. What kind of information you can take from your genome about your traits and whether it is actually correct. I guess it is just interesting how much the genome relates to everything.' ( $\mathrm{R} 8$, emphasis added)

None of our respondents disliked anything in particular in the testing process, with the exception of one who felt uncomfortable when she was contacted by other users of 23 andMe with similar genetic profiles:
'Someone contacted me and wrote "oh, we have seen your background and we could be relatives" or whatever, I don't remember, this I didn't like at all. So there is no way because we have similar backgrounds we may be relatives. [...] I blocked this person. But it is not his or her fault; it's that of 23andMe who allows this. But this is not Facebook. Certainly it is an important DNA information. If I wanted to have friends, I would just use Facebook you know. [...] I felt I had less control.' (R 7) 
Table 3. Overview of findings from qualitative data

Themes

(1) Motivation for undertaking DTC testing*

Curiosity

Scientific curiosity

Participation in research

Contributing to scientific advances of the future

Fun

Less interested in health-related results

(2) Experience with DTC testing*

Satisfied curiosity

Satisfied scientific curiosity

Gained familiarity with personal genomics

Gained experience with probabilistic data

Relative finder positive

Not concerned about negative results

Relative finder negative

Negative feedback from clinicians about test utility

(3) Recommendation of DTC to others*

Recommend because it is fun to do

Recommend because it encourages research participation

Recommend to those with genetic literacy

Do not recommend to people with anxieties

Recommend to adults only

No medical involvement required

Connected notions of scientific curiosity and research participation, data contribution as an investment for the future

Fun factor embedded in other motives

Limited expectations about the value of health-related results

Positive experience, fun factor, personal utility (for 3 categories)

Privacy concerns

Clinicians skeptical about clinical utility

Recommend for personal utility purposes, importance of research participation for future scientific advances

Concerns about potential negative reactions (for 3 categories)

Focus on personal utility rather than clinical utility

(4) Self-reported impact of testing*

No lifestyle changes

Led healthy lifestyle already

Pharmacogenomic use

(5) Concerns about DTC testing*

Potential privacy breaches

Concern about health insurance

Limited control of online privacy in general

Little attention to consent and privacy documents before

participating in testing

* Categories listed below.

Categories which were identified in the response of at least 5 respondents are in italics.

For another informant, in contrast, that very same feature at the 23andMe website - the 'Relative Finder' stood out as one of the most positive aspects of the testing process:

'It was cool [...] also to get this feedback from people. For example, one lady and one gentleman from the US contacted me saying we might be related, but they said they were not aware of any Swiss ancestry. [...] but many people with this background emigrated from an area very close to where my father comes from, in the 19th century. So we might be relatives. It was really: "oh cool".' (R 9)
Privacy key concern (for 2 categories)

Accept online privacy concerns as fact of modern life, concerns not prohibitive of participation

Limited impact (for all 3 categories) 
overly concerned, or to children. A decisive factor in deciding whether to recommend testing or not was the educational level of the would-be test-taker, in particular their level of genetic literacy [32].

Respondents said that the results did not have a significant impact on their lives or on their attitude towards their health. Most of them described themselves and their lifestyles as generally healthy. One informant said that some of the pharmagenomic information provided by 23 andMe could be useful in clinical practice, and another was able to link results to an existing condition s/he had. In relation to discussing results with a physician, most of the respondents found this 'unnecessary'. One informant, who had already discussed the results with her physician in the context of an existing condition, felt that the physician paid more attention to her as a result. And R 3 added that he was very surprised he got this information, he said, because it is usually quite expensive to do this SNP.

Others who discussed results with a physician did not find it helpful, particularly due to the physicians' negative reactions:

'The interesting thing is, I told my doctor I had taken this 23andMe test [in relation to pharmacogenomics] and he really thought this is not a serious thing.' (R 6)

'Actually a friend of ours is a medical doctor, and I discussed it with him, and he thought it was crazy altogether. It is completely useless for their practice, they think.' (R 10)

Like our questionnaire respondents, half of the informants acknowledged potential privacy concerns, but did not consider them prohibitive. They mentioned potential risks such as insurance companies obtaining or demanding access to such data, but they referred to this as an obstacle to be overcome. Participants acknowledged that there was limited control of privacy in every online environment, but none of them considered this a significant enough reason to prohibit the use of online genomic services:

'What I didn't like was the co-funding of 23andMe with Google. We are giving away too much responsibility to databases. [...] If you put it all together, bank information, genomics information, our communication, consumption and all that together, you might have a lot to lose.' (R 10)

'I don't think I am 100\% safe about [data] protection, if people are really curious about someone's data, they will find a thousand ways to get the information ...' (R 3)

'I am not concerned. Only my genetic data [alone] don't tell that much. If people want to know something [...], they can find it another way.' (R 5)

'Well, I am not so paranoid. [It is not that bad] if someone in the world knows that I am susceptible to developing diabetes ...' (R 6)

Early Users of Direct-to-Consumer

Genomics in Switzerland
The opportunity to participate in research, and the value of research in general, were prominent themes with all informants. In particular, the need for large data sets to catalyse progress in understanding the human genome was mentioned as one of the key reasons for their participation. Informants said that 23andMe gave them an opportunity they otherwise would not have had to help advance research. Individual contributions to research were discussed in terms of 'investments' in the future, with returns being the acceleration of medical discovery and better medicine. Perhaps also partly for this reason, most respondents said they had not read the informed consent document carefully. When asked why they had not, they said that they had a 'habit' of signing up to things online after taking just a quick look at the terms of conditions, and that they did not have any concerns about the research process. The demographics of the group might also further explain their ease with online platforms of any kind. Participants were aware of the privacy risks and the limited control of personal data once they were online; yet they seemed to accept this as a fact of modern life. It is notable that most of our interview participants had not spent much time reading the consent and privacy policies of $23 \mathrm{andMe}$, and none of them was aware of the Swiss expert committees' recommendation against online genetic testing.

\section{Discussion}

Participants both in the online questionnaire and in the interviews attributed limited significance to the extent to which test results were immediately relevant to their health. While there was interest in what kind of health information the test would yield, and how this information would be presented, even this 'professional' curiosity in health (given that almost all of our participants had life science degrees) was not mentioned as the decisive reason for taking the test. The driving factor for our informants was a more general - i.e. not health-focused - curiosity ranging from wanting to see 'what it looks like', to more specific technical aspects of how raw data are presented, how risk probability is derived in relation to published genome-wide association studies and what kind of research projects are available. Some respondents explicitly drew a distinction between scientific curiosity (or scientific interest) and general curiosity. Curiosity features as a motive in most other studies with users of DTC genomics testing services conducted to date. However, in those other studies, the main motiva-

Public Health Genomics 2012;15:352-362 
tion that participants stated for taking the test was the desire to learn about the degree to which they were at risk for developing particular conditions [13]. What we observed in our respondents, in contrast, was a much more general curiosity pertaining to technical aspects of the service. Health information was only the medium, not the target, of their interest.

Participants consistently referred to 'fun' when describing several aspects of the testing experience. The 'fun factor' manifested itself in different phases of the process, including the motivation for taking the test (participants thought of it as a fun thing to do), receiving the information (the actual experience was positive and pleasant) and putting the test results to use (either to share with friends and family or for research). The fact that most participants felt confident about evaluating the health information they received may partly explain why their focus was on enjoying other personal uses of the process, such as research participation. What was striking in the group we studied was that participants did not express worries about seeing results that could cause anxieties; this, however, may have to do with the professional background of virtually all the participants, who thus felt confident enough to understand and interpret the results. Moreover, it is likely that those who were concerned about the consequences of receiving SNP-based risk information did not sign up to order a test in the first place. In addition, a question arises as to whether the marketing messages of DTC companies inviting consumers to be in charge of their health might have been less appealing for our participants living outside the North American culture.

As highlighted earlier, the opportunity to participate in research was seen by the majority of our interview participants as an important aspect of the testing experience. For some participants, it was the main reason to get tested, while those who mentioned curiosity as the main motivation for taking the test saw research participation as an added benefit of the testing process; it enabled them to make a useful contribution to science and society. An altruistic motivation matching the profile of the 'health information altruist' [33] was coupled with the knowledge and conviction that large data sets are needed in order to successfully pursue genomic research. Moreover, the fact that many in our study group were professionally involved in research undoubtedly contributes to explaining this phenomenon.

In relation to the predictive power of the results, one of the themes that emerged in interactions with our study group was that of future utility. When talking about the information they received, many participants referred to the value that the results could have at a later stage. Actionability, therefore, was not understood to be immediate, but it expanded into the mid- or even long-term future. This extended also to the utility that our participants attributed to their participation in 23andMe's research endeavors promoted on the website. Getting involved in the present was seen as important in light of potential future benefits. This may help to explain (a) why our participants would recommend testing to family and friends; and (b) why they were comfortable with online access of testing without involvement on the part of medical professionals. While the concern about genetic literacy of lay people and its importance in interpreting results was appropriately raised, the overall moral imperative to foster scientific progress prevailed.

\section{Exploring Personal Utility}

Satisfaction of their (general or scientific) curiosity, and satisfaction/contentment from research participation, are both dimensions of personal utility, an aspect of personal genomics that has been insufficiently explored. Foster et al. [34] highlighted the need to expand the concept of utility in personal genomics beyond the narrow sense of clinical utility in order to encompass valuable uses that genomic information may have for the individual. Lesko et al. [35] further argued that the notion of clinical utility should be expanded to include personal utility. But even in these proposals, personal utility is implicitly assumed to relate to some future health goal(s). For example, individuals may use the (not immediately clinically actionable) information to change their lifestyle in order to improve their health.

Although these are steps in the right direction, these proposals do not go far enough $[36,37]$. What has emerged from our data is that facets of personal utility not directly linked to personal health outcomes played a prominent role in explaining why at least our study group engaged with DTC genomic testing. Our volunteers spoke of the test results - and in fact the entire testing experience - as being useful. What kind of utility is it that they were talking about, and why did it come up in connection with DTC genomics?

Other commentators have already alluded to the 'recreational' aspect of personal genomics and the interest of enthusiastic early adopters in participating in the 'genomic revolution' [38]. But such aspects have also been described as dangerous because they 'confuse entertainment with useful medical information' [39]. However, if users of DTC genomics services find the experience useful, in whatever way, on what basis should their claim to 
utility be overruled? In other words, in the context of testing that does not convey results with immediate clinical relevance, why does utility need to be defined in clinical terms? It is true that most of the existing DTC companies market their services as health-related; yet health is much broader than clinical medicine. Doing things that feel pleasant, that are 'fun', that make one engage with the genetic and lifestyle-related factors involved in health and disease, are related to the broader sense of well-being.

\section{Limitations}

This group is not representative of the broader population due to both their scientific expertise and their willingness to try out a new technology surrounded by controversy. As with early adopters of other technologies this group represents a group of ethical and cognitive 'pioneers' [40] because they are engaged with situations that both raise and address ethical and scientific questions not previously addressed in these ways. Their reasons for using DTC genomics and their attitude towards the process and its outcomes provide valuable insights into the moral and psychological background against which such technologies will be deployed.

Thus, although the specific characteristics of our participants prevent us from generalising our findings to wider groups, these same specific characteristics allow us to explore aspects of personal genomics which are likely to become of wider relevance. An example here is the notion of personal utility, which has been mentioned, but not yet conceptualised in the literature. Several questions remain unanswered and call for more research: would 'fun' and 'curiosity' be equally important motivators for other groups as they were for the group of people we studied? Are our findings specific to Switzerland and/or German-speaking regions? Larger empirical studies are needed in order to ascertain whether cultural differences in particular play a role in perceptions about DTC genomics and their moral acceptability.

\section{Conclusion}

In sum, we argue that the notion of personal utility requires further elaboration in order to take into account a broader spectrum of values, not merely clinical outcomes. Our study group, although being a very particular group due to the context in which they were recruited and their life science expertise, provided valuable insights into the alignment of personal curiosity and altruistic motivations (fostering medical research) and the utility attributed to both. A broader and more nuanced understanding of personal utility that makes room for a variety of aspects, including non-clinical aspects, needs to be developed. This will in turn enable us to better understand and evaluate the reasons that different groups have for engaging not only with DTC genetic testing but also with online-mediated health tools in general.

\section{Acknowledgments}

We would like to thank all study participants, the PHG anonymous reviewers for their insightful comments and Dr. Jonathan Ives for his input into an early version of the questionnaire.

\section{References}

$>1$ Harvey A, Brand A, Holgate ST, Kristiansen LV, Lehrach H, Palotie A, Prainsack B: The future of technologies for personalised medicine. New Biotechnol 2012;29:625-633.

2 Public Health Genomics European Network (PHGEN). http://www.phgen.eu.

-3 Evans JP, Green RC: Direct to consumer genetic testing: avoiding a culture war. Genet Med 2009; 11:568-569.

4 Bunnik EM, Schermer MH, Janssens AC: Personal genome testing: test characteristics to clarify the discourse on ethical, legal and societal issues. BMC Med Ethics 2011;12:11.

$\checkmark 5$ Evans JP, Dale DC, Fomous C: Preparing for a consumer-driven genomic age. $\mathrm{N}$ Engl J Med 2010;363:1099-1103.
6 Kuehn BM: Risks and benefits of direct-toconsumer genetic testing remain unclear. JAMA 2008;300:1503-1505.

$>7$ Patch C, Sequeiros J, Cornel MC: Genetic horoscopes: is it all in the genes? Points for regulatory control of direct-to-consumer genetic testing. Eur J Hum Genet 2009;17:857859.

8 Caulfield T: Direct-to-consumer genetics and health policy: a worst-case scenario? Am J Bioeth 2009;9:48-50.

$>9$ Gurwitz D, Bregman-Eschet Y: Personal genomics services: whose genomes? Eur J Hum Genet 2009; 17:883-889.

10 Mykitiuk R: Caveat emptor: direct-to-consumer supply and advertising of genetic testing. Clin Invest Med 2004;27:23-32.
11 Wilfond B, Ross LF: From genetics to genomics: ethics, policy, and parental decisionmaking. J Pediatr Psychol 2009;34:639-647.

12 Prainsack B, Wolinsky H: Direct-to-consumer genome testing: opportunities for pharmacogenomics research? Pharmacogenomics 2010;11:651-655.

13 Goldsmith L, Jackson L, O'Connor A, Skirton H: Direct-to-consumer genomic testing: systematic review of the literature on user perspectives. Eur J Hum Genet 2012;20: 811-816.

14 Bloss CS, Schork NJ, Topol EJ: Effect of direct-to-consumer genomewide profiling to assess disease risk. N Engl J Med 2011;364: 524-534. 


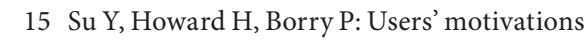
to purchase direct-to-consumer genomewide testing: an exploratory study of personal stories. J Community Genet 2011;2:135146.

16 Cherkas LF, Harris JM, Levinson E, Spector TD, Prainsack B: A survey of UK public interest in internet-based personal genome testing. PLoS One 2010;5:e13473.

17 Wright C, MacArthur DG: Direct to consumer genetic testing; in Best DH, Swensen JJ (eds): Molecular Genetics and Personalized Medicine - Molecular and Translational Medicine. New York, Springer, 2012.

18 Borry P, van Hellemondt RE, Sprumont D, Jales CF, Rial-Sebbag E, Spranger TM, Curren L, Kaye J, Nys H, Howard H: Legislation on direct-to-consumer genetic testing in seven European countries. Eur J Hum Genet 2012;20:715-721.

19 Google Insights: Insights for search. http:// www.google.com/insights/search/ (accessed May 22, 2012).

20 Academia Engelberg: Personalized Medicine: 10th dialogue on Science. September 14-16, 2011. http://www.academia-engelberg.ch/conference_2011.php5 (accessed May 22, 2012).

21 European Commission: eurostat: School enrolment and levels of education. http://epp. eurostat.ec.europa.eu/statistics_explained/ index.php/School_enrolment_and_early_ leavers_from_education_and_training (accessed October 10, 2012).

22 Federal Department of Foreign Affairs: People. http://www.swissworld.org/en/people/ language/english/ (accessed May 20, 2012).
23 Organisation for Economic Co-operation and Development (OECD): Country statistical profile: Switzerland 2011-2012. http:// www.oecd-ilibrary.org/economics/countrystatistical-profile-switzerland_20752288table-che (accessed May 22, 2012).

24 Swiss Biotech Association: Swiss Biotech Report 2012. http://www.swissbiotech.org/ home (accessed May 20, 2012).

25 Bundesgesetz über genetische Untersuchungen beim Menschen (GUMG): Article 5. http://www.admin.ch/ch/d/ff/2004/5483. pdf (accessed May 22, 2012).

26 Expertenkommission für genetische Untersuchungen beim Menschen (GUMEK): Informationskampagne: Gentests aus dem Internet 2009. http://www.bag.admin.ch/themen/medizin/00683/02724/04638/index. html?lang=de (accessed May 22, 2012).

-27 Gollust SE, Gordon ES, Zayac C, Griffin G, Christman MF, Pyeritz RE, Wawak L, Bernhardt BA: Motivations and perceptions of early adopters of personalized genomics: perspectives from research participants. Public Health Genomics 2012;15:22-30.

28 Ormond KE, Hudgins L, Ladd JM, Magnus DM, Greely HT, Cho MK: Medical and graduate students' attitudes toward personal genomics. Genet Med 2011;13:400-408.

29 Friese S: Qualitative Data Analysis with ATLAS.ti. London, Sage Publications, 2012.

30 Corbin J, Strauss AL, Juliet M: Grounded Theory in Practice. London, Sage Publications, 1997.
1 Eriksson N, Macpherson JM, Tung JY, Hon LS, Naughton B, Saxonov S, Avey L, Worcicki A, Pe'er I, Mountain J: Web-based, participant-driven studies yield novel genetic associations for common traits. PLoS Genet 2010;6:e1000993.

32 Sørensen K, Brand H: Health literacy: the essential catalyst for the responsible and effective translation of genome-based information for the benefit of population health. Public Health Genomics 2011;14:195-200.

33 Kohane IS, Altman RB: Health-information altruists - a potentially critical resource. $\mathrm{N}$ Engl J Med 2005;353:2074-2077.

34 Foster MW, Mulvihill JJ, Sharp RR: Evaluating the utility of personal genomic information. Genet Med 2009;11:570-574.

35 Lesko LJ, Zineh I, Huang SM: What is clinical utility and why should we care? Clin Pharmacol Ther 2010;88:729-733.

36 Brand A: Public health genomics - public health goes personalized? Eur J Public Health 2011;21:2-3.

- 37 Cesuroglu T, Ommen B, Malats N, Sudbrak $\mathrm{R}$, Lehrach $\mathrm{H}$, Brand A: Public health perspective: from personalized medicine to personal health. Pers Med 2012;9:115-119.

38 Van Ommen GB, Cornel MC: Recreational genomics? Dreams and fears on genetic susceptibility screening. Eur J Hum Genet 2008; 16:403-404.

39 Evans JP: Recreational genomics; what's in it for you? Genet Med 2008;10:709-710.

40 Rapp R: Testing women, testing the fetus. London, Routledge, 1999. 